\title{
Mr. Paint and Mrs. Correct
}

\author{
Uwe Schauz \\ Department of Mathematics and Statistics \\ King Fahd University of Petroleum and Minerals \\ Dhahran 31261, Saudi Arabia \\ schauz@kfupm.edu.sa
}

Submitted: Jul 21, 2008; Accepted: Jun 19, 2009; Published: Jun 25, 2009

Mathematics Subject Classifications: 91A43, 05C15, 05C20, 05C10

\begin{abstract}
We introduce a coloring game on graphs, in which each vertex $v$ of a graph $G$ owns a stack of $\ell_{v}-1$ erasers. In each round of this game the first player Mr. Paint takes an unused color, and colors some of the uncolored vertices. He might color adjacent vertices with this color - something which is considered "incorrect". However, Mrs. Correct is positioned next to him, and corrects his incorrect coloring, i.e., she uses up some of the erasers - while stocks (stacks) last - to partially undo his assignment of the new color. If she has a winning strategy, i.e., she is able to enforce a correct and complete final graph coloring, then we say that $G$ is $\ell$-paintable.

Our game provides an adequate game-theoretic approach to list coloring problems. The new concept is actually more general than the common setting with lists of available colors. It could have applications in time scheduling, when the available time slots are not known in advance. We give an example that shows that the two notions are not equivalent; $\ell$-paintability is stronger than $\ell$-list colorability. Nevertheless, many deep theorems about list colorability remain true in the context of paintability. We demonstrate this fact by proving strengthened versions of classical list coloring theorems. Among the obtained extensions are paintability versions of Thomassen's, Galvin's and Shannon's Theorems.
\end{abstract}

\section{Introduction}

There are many papers about graph coloring games. Originally, these games were introduced with the aim to provide a game-theoretic approach to coloring problems. The hope was to obtain good bounds for the chromatic number of graphs, in particular with regards to the Four Color Problem (see, e.g., [BGKZ] and the literature cited there). However, there is a fundamental problem with these games, which means that they cannot fulfill 
their original purpose. Typically, these games require many more colors than those actually needed for a correct graph coloring, so there is a large gap between the corresponding game chromatic numbers and the chromatic number. Hence, even best possible upper bounds for these game chromatic numbers are usually bad upper bounds for the chromatic or the list chromatic number, i.e., the minimal size of given color lists $L_{v}$, assigned to the vertices $v$ of a graph $G$, which ensures the existence of a correct vertex coloring $\lambda: v \longmapsto \lambda_{v} \in L_{v}$ of $G$. (See $[\mathrm{Al}],[\mathrm{Tu}]$ and $[\mathrm{KTV}]$ in order to get an overview of list colorings.)

The game of Mr. Paint and Mrs. Correct, introduced in Section 1 (in Game 1.1 and its reformulation Game 1.6), is different. It provides an adequate game-theoretic approach to list coloring problems. The existence of a winning strategy for Mrs. Correct, which we call $\ell$-paintability (see Definition 1.2 or the reformulated recursive Definition 1.8), comes very close to $\ell$-list colorability (Definition 1.3). The $\ell$-paintability is stronger than the $\ell$-list colorability (Preposition 1.4), but not by much. Although Example 1.5 shows that there is a gap between these two notions, most theorems about list colorability hold for paintability as well. Therefore, good bounds for the painting number - which may be found using game-theoretic approaches - are usually good bounds for the list chromatic number as well. The reason for all this is that (as described after Definition 1.3) paintability can be seen as a dynamic version of list colorability, where the lists of colors are not completely fixed before the coloring process starts. Beyond this connection to list colorings, paintability also may have interesting new applications. See [Scha2, Example 3.11] for an application to a time scheduling problem that demonstrates the advantage of the new painting concept against the list coloring approach with fixed list of available time slots.

All list coloring theorems - whose proofs are exclusively based on coloring extension techniques, on the existence of kernels, and on Alon and Tarsi's Theorem - can be transferred into a paintability version. These three techniques are the main techniques in the theory of list colorings. In addition, for colorings in the classical sense, there is the important recoloring technique (Kempe-chain technique). It is used for example in the proofs of Vizing's Theorem, and works with neither list colorings nor with paintability.

In Section 2 we prove several lemmas that can be used as a replacement for coloring extension techniques. They are based on a technique, called the pre-use of additional erasers, which is described in Preposition 2.1. We demonstrate the application of these replacements in the proof of Theorem 2.6, a strengthening of Thomassen's Theorem about the 5-list colorability of planar graphs.

In Section 3 (Lemma 3.1), we strengthen Bondy, Boppana and Siegel's Kernel Lemma. Afterwards, we apply it in the proof of Galvin's celebrated theorem about the list chromatic index of bipartite graphs (Theorem 3.2), and in Borodin, Kostochka and Woodall's strengthening of Galvins's result (Theorem 3.3). This leads also to a strengthening of their refinement of Shannon's bound for the list chromatic index of multigraphs (Theorem 3.5).

We are also working [Scha2] on a purely combinatorial proof of a paintability version of Alon and Tarsi's Theorem [AlTa] about colorings and orientations of graphs. This will lead 
to paintability versions of many other list coloring theorems, e.g., Alon and Tarsi's bound of the list chromatic number of bipartite and planar bipartite graphs, and Häggkvist and Janssen's bound for the list chromatic index of the complete graph $K_{n}$. Brooks' Theorem can be strengthened as well using the Alon-Tarsi-Theorem. Our version will even be stronger than the version of Borodin and of Erdős, Rubin and Taylor. Furthermore, we will present in [Scha3] a paintability version of the Combinatorial Nullstellensatz [Al2, Scha1], and will apply it to hypergraphs.

\section{Mr. Paint and Mrs. Correct}

The game of Mr. Paint and Mrs. Correct is a game with complete information, played on a fixed given graph $G=(V, E)$. It is defined as follows:

Game 1.1 (Paint-Correct-Game). Mr. Paint has many different colors, at least one for each round of the game. In each round he uses a new color that cannot be used again. Mrs. Correct has a finite stack $S_{v}$ of erasers for each vertex $v \in V$ of the underlying graph $G$. They are lying at the corresponding vertices, ready for use.

The game of Mr. Paint and Mrs. Correct works as follows:

1P: Mr. Paint starts, and in the first round he uses his first color to color some (at least one) vertices of $G$.

1C: Mrs. Correct may use - and hereby use up - for each newly colored vertex $v$ one eraser from $S_{v}$ (if $\left.S_{v} \neq \varnothing\right)$ to clear $v$. It is the job of Mrs. Correct to avoid monochromatic edges, i.e., edges with ends of the same color.

2P: In the second round Mr. Paint uses his second color to color some (at least one) of the by now uncolored vertices of $G$.

2C: Mrs. Correct, again, uses up erasers from some stacks $S_{v}$ belonging to the newly colored vertices $v$, to avoid monochromatic edges.

$$
\vdots \quad \vdots
$$

End: The game ends when one player cannot move anymore, and hence loses.

Mrs. Correct cannot move if not enough erasers are available with which she could avoid monochromatic edges, so that the remaining partial coloring would be incorrect.

Mr. Paint loses if all vertices have already been colored when it is his turn.

This game ends after at most $\sum_{v \in V}\left(\left|S_{v}\right|+1\right)$ rounds. If Mrs. Correct wins, then the game results in a proper coloring of $G$. In this case, Mrs. Correct has rejected the color of each vertex $v \in V$ up to $\left|S_{v}\right|$ times. Put another way, we could imagine that Mr. Paint 
uses real paint and varnishes the vertices with it, and that Mrs. Correct uses sandpaper pieces to roughen the paint surface. In this way we obtain up to $\ell_{v}:=\left|S_{v}\right|+1$ layers of paint on each $v \in V$, which leads us to the following terminology:

Definition 1.2 (Paintability). Let $\ell=\left(\ell_{v}\right)_{v \in V}$ be defined by $\ell_{v}:=\left|S_{v}\right|+1$. If there is $\ell, \ell_{v}$ a winning strategy for Mrs. Correct, then we say that $G$ is $\ell$-paintable. We also say that $G^{\ell}$ is paintable, where $G^{\ell}$ is the graph $G$ together with $\ell_{v}-1$ erasers at each vertex $v \in V$ (the mounted graph, as we call it).

We write $n$-"something" instead of $(n 1)$ - "something", where $1=(1)_{v \in V}$ and $n \in \mathbb{N}$.

There is a connection to list colorings, which are defined as follows:

Definition 1.3 (List Colorings). A product $L=\prod_{v \in V} L_{v}$ of sets $L_{v}$ (called lists) of $\ell_{v}$ $L, L_{v}$ elements (called colors) is an $\ell$-product (where $\ell:=\left(\ell_{v}\right)_{v \in V}$ ).

If there is a (proper) coloring $\lambda \in L$ of $G$ - i.e., if $\lambda_{u} \neq \lambda_{v}$ for all $u v \in E$ - then we say that $G$ is $L$-colorable. If $G$ is $L$-colorable for all $\ell$-products $L$, then we say that $G$ is $\ell$-list colorable or just $\ell$-colorable.

Imagine that Mr. Paint writes down the colors he suggests for the vertex $v$ in a list $L_{v}$. At the end of the game the list $L_{v}$ has at most $\ell_{v}:=\left|S_{v}\right|+1$ entries, since $\left|S_{v}\right|$ is the maximal number of rejections at $v$. Furthermore, if $v$ "wears" a color at the end of the game, then its color lies in the list $L_{v}$. Hence, paintability may be seen as a dynamic version of list colorability, where the lists $L_{v}$ are not completely fixed before the coloration process starts. Thus we have the following connection to the usual list colorability:

Proposition 1.4. Let $G$ be a graph and $\ell \in \mathbb{N}^{V}$.

$$
G \text { is } \ell \text {-paintable. } \Longrightarrow G \text { is } \ell \text {-list colorable. }
$$

The following example shows the strictness of this statement:

Example 1.5. The graph $G$ in Figure 1 below is $\ell$-list colorable but not $\ell$-paintable, where $\ell_{v}:=2$ for all vertices $v \in V$ except the center $v_{5}$, for which $\ell_{v_{5}}:=3$ :

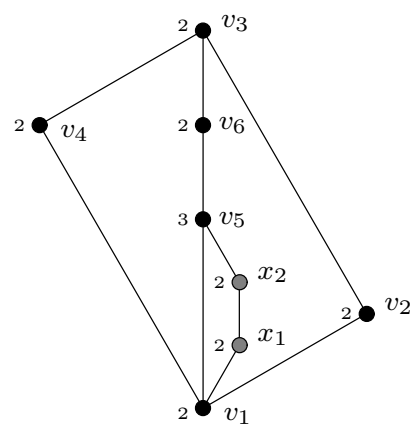

Figure 1: An $\ell$-list colorable but not $\ell$-paintable graph. 
Proof. We start with the unpaintability of $G$ : In order to prevail, Mr. Paint colors the vertices $x_{1}$ and $x_{2}$ in his first move. If Mrs. Correct then clears $x_{1}$, Mr. Paint can win as the induced subgraph $G\left[x_{1}, v_{1}, v_{2}, v_{3}, v_{4}\right]$ is not even $L$-colorable for

$$
L=L_{x_{1}} \times L_{v_{1}} \times L_{v_{2}} \times L_{v_{3}} \times L_{v_{4}}:=\{1\} \times\{1,2\} \times\{2,3\} \times\{3,4\} \times\{4,2\} .
$$

Indeed, this argument shows that the whole remaining uncolored part $G \backslash x_{2}$ of $G$ is not list colorable for updated list sizes; and uncolorability implies unpaintability, as we have seen in Proposition 1.4. Thus, Mrs. Correct cannot find a strategy for the remaining uncolored part $G \backslash x_{2}$ of $G$. (See also the recursive description of the game below).

If Mrs. Correct sands off $x_{2}$, then Mr. Paint can win for the same reason. In this case there is an odd circuit in the remaining uncolored part $G \backslash x_{1}$ which cannot be colored with 2 colors, and the third color of $v_{5}$ can be "neutralized" through its neighbor $x_{2}$. Summarizing, Mr. Paint wins in any case, and $G$ is not $\ell$-paintable. If

We come now to the $\ell$-list colorability, and have to examine all possible $\ell$-products $L$ :

$$
L_{x_{1}}=L_{x_{2}} \quad \text { or } \quad L_{x_{1}} \cap L_{x_{2}}=\varnothing
$$

then each proper coloring of $G \backslash\left\{x_{1}, x_{2}\right\}$ extends to a proper coloring of $G$. It is thus sufficient to examine the more difficult case:

$$
L_{x_{1}}:=\{1,2\} \quad \text { and } \quad L_{x_{2}}:=\{2,3\} .
$$

In this case we have to find a coloring $\lambda$ of $G \backslash\left\{x_{1}, x_{2}\right\}$ with

$$
\left(\lambda_{v_{1}}, \lambda_{v_{5}}\right) \neq(1,3) \text {. }
$$

If, for example, there is a coloring $\lambda$ of the path $v_{1} v_{2} v_{3} v_{4}$ with

$$
\lambda_{v_{4}} \neq \lambda_{v_{1}} \neq 1
$$

then this partial coloring can be extended to $v_{6}$, then to $v_{5}$ and finally to the whole graph $G$. However, such extendable colorings of the path $v_{1} v_{2} v_{3} v_{4}$ always exist, except when the lists to $v_{1}, v_{2}, v_{3}$ and $v_{4}$ have the following "chain structure":

$$
L_{v_{1}} \times L_{v_{2}} \times L_{v_{3}} \times L_{v_{4}}:=\{1, a\} \times\{a, b\} \times\{b, c\} \times\{c, a\} \quad \text { where } \quad a \neq b \neq c \neq a .
$$

But then we can choose

$$
\lambda_{v_{4}}:=a, \quad \lambda_{v_{1}}:=1 \quad \text { and } \quad \lambda_{v_{2}}:=a,
$$

and this partial coloring is extendable, at first to $v_{5}$, with $\lambda_{v_{5}} \neq 3$, then to $x_{1}, x_{2}$ and to $v_{6}$, and finally to $v_{3}$, which still has the two colors $b \neq a$ and $c \neq a$ "available".

Now, we come to a more recursive formulation of our game, which is more easily accessible for proofs by induction. It is based on the simple observation that - since Mr. Paint uses an extra color for each round - it makes no difference whether one looks for coloring extensions of the partially colored graph $G$, or whether one cuts off the already colored vertices from the graph and colors the remaining graph. More precisely, we have the following reformulation: 
Game 1.6 (Reformulation). In this reformulation Mr. Paint has just one marker. As this is his only possession some call him Mr. Marker, but that is just a nickname.

Mrs. Correct has a finite stack $S_{v}$ of erasers for each vertex $v$ in $G_{1}:=G$. They are lying on the corresponding vertices, ready for use.

The reformulated game of Mr. Paint and Mrs. Correct works as follows:

1P: Mr. Paint starts, choosing a nonempty set of vertices $V_{1 P} \subseteq V\left(G_{1}\right)$ and marking them with his marker.

1C: Mrs. Correct chooses an independent subset $V_{1 C} \subseteq V_{1 P}$ of marked vertices in $G_{1}$, i.e., $u v \notin E\left(G_{1}\right)$ for all $u, v \in V_{1 C}$. She cuts off the vertices in $V_{1 C}$, so that the graph $G_{2}:=G_{1} \backslash V_{1 C}$ remains. The still marked vertices $v \in V_{1 P} \backslash V_{1 C}$ of $G_{2}$ have to be cleared. Therefore, Mrs. Correct must use one eraser from each of the corresponding stacks $S_{v}$. She loses if she runs out of erasers and cannot do that, i.e., if already $S_{v}=\varnothing$ for a still marked vertex $v \in V_{1 P} \backslash V_{1 C}$.

2P: Mr. Paint again chooses a nonempty set of vertices $V_{2 P} \subseteq V\left(G_{2}\right)$ and marks them with his marker.

$2 C$ : Mrs. Correct again cuts off an independent set $V_{2 C} \subseteq V_{2 P}$, so that a graph $G_{3}:=$ $G_{2} \backslash V_{2 C}$ remains. She also uses (and uses up) some erasers to clear the remaining marked vertices $v \in V_{2 P} \backslash V_{2 C}$.

End: The game ends when one player cannot move anymore, and hence loses.

Mrs. Correct cannot move if she does not have enough erasers left to clear the vertices she was not able to cut off.

Mr. Paint loses if there are no more vertices left.

With this reformulation the original Definition 1.2 of paintability can be rewritten. At first, we introduce an appropriate notation for the graphs $G_{1}, G_{2}, \ldots$, produced in this version of the game, and their corresponding mounted graphs. Using characteristic maps/tuples of subsets $U \subseteq V$ and of elements $u \in V$, namely

$$
1_{U}:=\left(?_{(v=U)}\right)_{v \in V} \in\{0,1\}^{V} \quad \text { and } \quad 1_{u}:=1_{\{u\}},
$$

based on the "Kronecker query" ? ${ }_{(\mathcal{A})}$, defined for statements $\mathcal{A}$ by

$$
?_{(\mathcal{A})}:= \begin{cases}0 & \text { if } \mathcal{A} \text { is false } \\ 1 & \text { if } \mathcal{A} \text { is true }\end{cases}
$$

we provide: 
Definition 1.7. Let $G^{\ell}$ be a mounted graph. We treat $G^{\ell}$ as any usual graph; but, when we change the graph, we adapt the stacks of erasers in the natural way. For example we set for sets $U$ of vertices and edges

$$
G^{\ell} \backslash U:=(G \backslash U)^{\left.\ell\right|_{V \backslash U}}
$$

We also introduce a new operation $l($ down $)$ which acts only on the stacks of erasers:

$$
G^{\ell} \downarrow U:=G^{\ell-1_{(U \cap V)}} .
$$

Now, the remaining graph $G_{2}$, after Mrs. Correct's first move $1 C$, together with the remaining stacks of reduced sizes

$$
\ell_{v}^{2}-1 \leq \ell_{v}^{1}-1:=\ell_{v}-1 \text { for all } v \in V
$$

can be written as:

$$
G_{2}^{\ell^{2}}=G_{1}^{\ell^{1}} \backslash V_{1 C} \downarrow V_{1 P}
$$

Furthermore, we obtain a handy recursive definition for paintability:

Definition 1.8 (Paintability - Reformulation). For $\ell \in \mathbb{N}^{V}$ the $\ell$-paintability of $G$, i.e., the paintability of $G^{\ell}$, can be defined recursively as follows:

(i) $G=\varnothing$ is $\ell$-paintable (where $V=\varnothing$ so that $\ell$ is the empty tuple).

(ii) $G \neq \varnothing$ is $\ell$-paintable if $\ell \geq 1$ and if each nonempty subset $V_{P} \subseteq V$ of vertices contains a good subset $V_{C} \subseteq V_{P}$, i.e., an independent set $V_{C} \subseteq V_{P}$, such that $G^{\ell} \backslash V_{C} \downarrow V_{P}$ is paintable.

It is obvious, that if $V_{C} \subseteq U \subseteq V_{P}$ and $V_{C}$ is good in $V_{P}$, then $V_{C}$ is also good in $U$. If, in addition, $U$ is independent, then $U$ is good in $V_{P}$. Conversely, in Proposition 2.1 we will learn that, if $V_{C}$ is good in $U$, then $V_{C}$ is also good in $V_{P} \supseteq U$, but for the price of additional erasers, i.e. if we put one additional eraser on each vertex $v$ of $V_{P} \backslash U$. This will be important when we generalize theorems, based on coloring extension techniques, to paintability.

Before we come to this, we want to mention that, with slight modifications that do not affect the definition of paintability, our game can be viewed as a game in the sense of Conway's game theory [Co], [SSt]. From this point of view, graphs are not just either $\ell$-paintable or not $\ell$-paintable, but some graphs may be more $\ell$-paintable than others. However, this game is not a "cold" game, i.e., it is usually no number. 


\section{Coloring Extensions and Cut Lemmas}

In this section we generalize coloring extension techniques to paintability. When we try to find list colorings, we may choose a particular vertex enumeration $v_{1}, v_{2}, \ldots, v_{n}$, and color the vertices $v_{i}$ in turn, with a color not used for any neighbor of $v_{i}$ among the successors $v_{1}, v_{2}, \ldots, v_{i-1}$. This technique cannot be used in the frame of paintability, but the following lemmas can provide a replacement. These replacements are then used at the end of the section to prove a strengthening of Thomassen's Theorem. Note that the corresponding list coloring versions of the used lemmas are almost trivial.

The proofs of the lemmas are based on a technique that we call pre-use of additional erasers. It means that additional erasers can be used before one has to look after a winning move. More exactly:

Proposition 2.1 (Pre-Usage Argument). Let $G^{\ell}$ be a mounted graph, and assume that $M r$. Paint has marked a subset $V_{P} \subseteq V$, in which Mrs. Correct should find a good subset $V_{C} \subseteq V_{P}$. If we put additional erasers on the vertices of a subset $U \subseteq V_{P}$, then Mrs. Correct may use the additional erasers at first, and then search for a good subset in $V_{P} \backslash U$ :

If $V_{C}$ is good in the remaining set $V_{P} \backslash U$, with respect to $\ell$,

then $V_{C}$ is also good in $V_{P}$, but with respect to $\ell+1_{U}$.

More general, for arbitrary subsets $U, V_{C}, V_{P} \subseteq V$, the following equality holds:

$$
G^{\ell+1\left(U \cap V_{P}\right)} \backslash V_{C} \downarrow V_{P}=G^{\ell} \backslash V_{C} \downarrow\left(V_{P} \backslash U\right) .
$$

Lemma 2.2 (Edge Lemma). Let two different vertices $u$ and $w$ of $G$ be given. The $\ell$-paintability of $G$ implies the $\left(\ell+\ell_{w} 1_{u}\right)$-paintability of $G \cup w u:=(V, E \cup\{w u\})$.

Proof. Let a nonempty subset $V_{P} \subseteq V$ be given. If $w \in V_{P}$, we pre-use one additional eraser, and choose

$$
V_{C} \operatorname{good} \text { in } \quad V_{P} \backslash u:=V_{P} \backslash\{u\}
$$

with respect to $\ell$ and $G$. Using Preposition 2.1, we know that

$$
V_{C} \text { is also good in } V_{P}
$$

but with respect to $\ell+1_{u}$ and $G$.

If now $w \notin V_{C}$, then we apply an induction argument to

$$
G^{\prime \ell^{\prime}}:=G^{\ell+1_{u}} \backslash V_{C} \downarrow V_{P},
$$

which has one eraser fewer at $w \in V_{P}$, i.e.,

$$
\ell_{w}^{\prime}=\ell_{w}-1 .
$$

It follows the paintability of

$$
\left(G^{\prime} \cup w u\right)^{\ell^{\prime}+\ell_{w}^{\prime} 1_{u}} \stackrel{(17)}{=}\left(G^{\ell+1_{u}+\ell_{w}^{\prime} 1_{u}} \backslash V_{C} \downarrow V_{P}\right) \cup w u=(G \cup w u)^{\ell+\ell_{w} 1_{u}} \backslash V_{C} \downarrow V_{P},
$$


so that the recursive Definition 1.8 applies and accomplishes this case.

If $w \in V_{C}$ then exactly one end of $w u$ lies in $V_{C}$ (since we chose $V_{C} \subseteq V_{P} \backslash u$ ),

$$
(G \cup w u) \backslash V_{C}=G \backslash V_{C},
$$

and

$$
(G \cup w u)^{\ell+1_{u}} \backslash V_{C} \downarrow V_{P}=G^{\ell+1_{u}} \backslash V_{C} \downarrow V_{P}
$$

is still paintable, so that

$$
V_{C} \text { is good in } V_{P}
$$

even with respect to $G \cup w u$ and $\ell+1_{u} \leq \ell+\ell_{w} 1_{u}$.

If $w \notin V_{P}$ things are even simpler, we choose

$$
V_{C} \operatorname{good} \text { in } V_{P}
$$

with respect to $\ell$ and $G$; i.e., $G^{\ell} \backslash V_{C} \downarrow V_{P}$ is paintable. If, now, $u \in V_{C}$ then again exactly one end of $w u$ lies in $V_{C}$ and we can argue as above. In the other case we use an induction argument to prove the paintability of $(G \cup w u)^{\ell+\ell_{w} 1_{u}} \backslash V_{C} \downarrow V_{P}$, and apply Definition 1.8.

Later on in this paper we will need the following simple lemma, which can also be applied to single vertices (the case $|U|=1$ as well as the case $|W|=1$ ):

Lemma 2.3 (Cut Lemma). Let $V=U \uplus W$ (disjoined union) be a partition of the vertex set of $G$, and let $\eta_{u}:=|N(u) \cap W|$ be the number of neighbors of $u \in U$ in $W$.

If $G[U]$ is $\ell_{U}$-paintable and $G[W]$ is $\ell_{W}$-paintable then $G$ is $\left(\ell_{U}+\ell_{W}+\eta\right)$-paintable; where $\eta:=\left(\eta_{u}\right)_{u \in U}$, and where this $\eta$, as well as $\ell_{U}$ and $\ell_{W}$, is "filled up" with zeros, in order to view it as a tuple over $V$.

Proof. Let a nonempty subset $V_{P} \subseteq V$ be given, and choose

$$
W_{C} \text { good in } W_{P}:=V_{P} \cap W
$$

with respect to $\ell_{W}$ and $G[W]$. Now, let $N\left(W_{C}\right)$ be the set of all neighbors of vertices in $W_{C}$. We pre-use the erasers in the subset

$$
\Delta:=V_{P} \cap U \cap N\left(W_{C}\right) \subseteq V_{P} \cap U
$$

and choose

$$
U_{C} \quad \text { good in } \quad U_{P}:=V_{P} \cap U \backslash N\left(W_{C}\right)
$$

with respect to $\ell_{U}$ and $G[U]$; i.e., using Preposition 2.1, we know that

$$
U_{C} \quad \text { is also good in } \quad V_{P} \cap U=U_{P} \uplus \Delta
$$

but with respect to $\ell_{U}+1_{\Delta}$ and $G[U]$. In other words, if we introduce the set

$$
V_{C}:=U_{C} \uplus W_{C},
$$


the mounted graphs

$$
G[W]^{\ell_{W}} \backslash W_{C} \downarrow\left(V_{P} \cap W\right)=\left(G^{\ell_{W}} \backslash V_{C} \downarrow V_{P}\right)\left[W \backslash W_{C}\right]
$$

and

$$
G[U]^{\ell_{U}+1_{\Delta}} \backslash U_{C} \downarrow\left(V_{P} \cap U\right)=\left(G^{\ell_{U}+1_{\Delta}} \backslash V_{C} \downarrow V_{P}\right)\left[U \backslash U_{C}\right]
$$

are paintable, and an induction argument implies that

$$
\left(G^{\ell_{W}+\ell_{U}+1_{\Delta}+\eta^{\prime}} \backslash V_{C} \downarrow V_{P}\right)\left[V \backslash V_{C}\right]=G^{\ell_{W}+\ell_{U}+1_{\Delta}+\eta^{\prime}} \backslash V_{C} \downarrow V_{P}
$$

is paintable as well, where

$$
\eta_{u}^{\prime}:=\left|N(u) \cap W \backslash W_{C}\right| \text { for all } u \in U .
$$

Since neighbors $u$ of elements $w \in W_{C}$ have fewer neighbors in $W \backslash W_{C}$ than in $W$

$$
\eta_{u}^{\prime}<\eta_{u} \quad \text { for all } u \in N\left(W_{C}\right)
$$

and

$$
\eta^{\prime}+1_{\Delta} \leq \eta
$$

It follows that

$$
G^{\ell_{W}+\ell_{U}+\eta} \backslash U_{C} \downarrow V_{P}
$$

is paintable, so that the recursive Definition 1.8 applies.

Lemma 2.3 does not suffice to prove Thomassen's Theorem 2.6. We will need the following version of its $|W|=1$ case, which requires more additional erasers, but also saves one at one distinguished neighbor $u_{0}$ of $w$ :

Lemma 2.4 (Vertex Lemma). Let $w u_{0} \in E$ be given and set $\eta_{w}:=2, \eta_{u_{0}}:=0, \eta_{u}=2$ for all other neighbors $u$ of $w$, and $\eta_{v}=0$ for the remaining vertices $v$ of $G$.

If $G \backslash w$ is $\ell$-paintable then $G$ is $(\ell+\eta)$-paintable; where $\eta:=\left(\eta_{v}\right)_{v \in V}$, and where $\ell \in \mathbb{N}^{V \backslash w}$ is "filled up" with one zero $\left(\ell_{w}:=0\right)$, in order to view it as tuple over $V$.

Proof. Let a nonempty subset $V_{P} \subseteq V$ be given. Using an induction argument, as in the last part of the proof of Lemma 2.2, we may suppose that $w \in V_{P}$. Let

$$
N:=\left\{u \neq u_{0} \quad \mid \operatorname{dist}(u, w) \leq 1\right\}
$$

and choose

$$
V_{C}^{\prime} \operatorname{good} \text { in } \quad V_{P}^{\prime}:=V_{P} \backslash N
$$

with respect to $\ell$ and $G \backslash w$; i.e.,

$$
(G \backslash w)^{\ell} \backslash V_{C}^{\prime} \downarrow V_{P}^{\prime}
$$

is paintable. Of course, we want to apply a pre-usage argument to the difference

$$
V_{P} \backslash V_{P}^{\prime}=V_{P} \cap N \text {. }
$$


We distinguish two cases:

If $u_{0} \in V_{C}^{\prime}$ we apply Lemma 2.3 to $G^{\ell+1_{w}} \backslash V_{C}^{\prime} \backslash V_{P}^{\prime}$, where we choose $W:=\{w\}$, $U:=(V \backslash w) \backslash V_{C}^{\prime}$ and use the inherited stacks, e.g., $\ell_{W}:=1_{w}$. It follows that

$$
G^{\ell+\eta^{\prime}} \backslash V_{C}^{\prime} \downarrow V_{P}^{\prime}=G^{\ell+\eta^{\prime}+1_{\left(V_{P} \cap N\right)}} \backslash V_{C}^{\prime} \downarrow V_{P}
$$

is paintable; where $\eta_{w}^{\prime}:=1, \eta_{u}^{\prime}:=1$ for all neighbors $u$ of $w$ in $G \backslash V_{C}^{\prime}$, and $\eta_{v}^{\prime}:=0$ for the remaining vertices $v$ of $G$. As we assumed $u_{0} \in V_{C}^{\prime}$ this means that $\eta_{u_{0}}^{\prime}=0$ and hence

$$
\eta^{\prime}+1_{\left(V_{P} \cap N\right)} \leq \eta
$$

so that

$$
V_{C}^{\prime} \text { is good in } V_{P}
$$

with respect to $\ell+\eta$ and $G$.

If $u_{0} \notin V_{C}^{\prime}$ then, on one hand, $w$ has no neighbor in $V_{C}^{\prime}$, and $V_{C}^{\prime} \cup\{w\}$ is independent in $G$, on the other hand, as we have seen above,

$$
G^{\ell+1_{\left(V_{P} \cap N\right)}} \backslash\left(V_{C}^{\prime} \cup\{w\}\right) \downarrow V_{P}=(G \backslash w)^{\ell} \backslash V_{C}^{\prime} \downarrow V_{P}^{\prime}
$$

is paintable. Hence,

$$
V_{C}^{\prime} \cup\{w\} \text { is good in } V_{P}
$$

with respect to $G$ and $\ell+\eta \geq \ell+1_{\left(V_{P} \cap N\right)}$.

We will also need the following lemma that, together with the Edge Lemma 2.2, could be used in another proof of the Cut Lemma 2.3:

Lemma 2.5 (Merge Lemma). Let $G^{\ell}:=G^{\prime \ell^{\prime}} \cup G^{\prime \prime \ell^{\prime \prime}}$ be the union $G^{\prime} \cup G^{\prime \prime}$ of two graphs $G^{\prime \ell^{\prime}} \cup G^{\prime \prime \ell^{\prime \prime}}$ $G^{\prime}$ and $G^{\prime \prime}$, together with the inherited erasers, i.e.,

$$
\ell-1:=\left(\ell^{\prime}-1\right)+\left(\ell^{\prime \prime}-1\right)
$$

where $\ell^{\prime}-1$ and $\ell^{\prime \prime}-1$ are "filled up" with zeros, in order to view them as tuples over the set $V$. Suppose further that in $G^{\prime \prime}$ there are no erasers at the vertices of the intersection, i.e.,

$$
\left.\ell^{\prime \prime}\right|_{U} \equiv 1, \quad \text { where } \quad U:=V\left(G^{\prime}\right) \cap V\left(G^{\prime \prime}\right) .
$$

If $G^{\prime \ell^{\prime}}$ and $G^{\prime \prime \ell^{\prime \prime}}$ are paintable, then $G^{\ell}:=G^{\prime \ell^{\prime}} \cup G^{\prime \ell^{\prime \prime}}$ is paintable as well.

Proof. In order to prove the paintability of $G^{\ell}$, we have to find a good subset $V_{C}$ in each fixed given nonempty subset $V_{P} \subseteq V$. To this end, we choose

$$
V_{C}^{\prime} \quad \text { good in } \quad V_{P}^{\prime}:=V_{P} \cap V\left(G^{\prime}\right)
$$

with respect to $G^{\prime \ell^{\prime}}$, and we choose

$$
V_{C}^{\prime \prime} \quad \operatorname{good} \text { in } \quad V_{P}^{\prime \prime}:=\left(V_{P} \backslash V\left(G^{\prime}\right)\right) \uplus\left(U \cap V_{C}^{\prime}\right)
$$


with respect to $G^{\prime \prime \ell^{\prime \prime}}$. Since no erasers lie at the vertices $u \in U \cap V_{P}^{\prime \prime}$ of $G^{\prime \prime}$, they have to be cut off, i.e.,

$$
U \cap V_{P}^{\prime \prime} \subseteq V_{C}^{\prime \prime} \subseteq V_{P}^{\prime \prime}
$$

Moreover, intersecting these sets with $U$, we see that

$$
U \cap V_{C}^{\prime \prime}=U \cap V_{P}^{\prime \prime} \stackrel{(48)}{=} U \cap V_{C}^{\prime}
$$

Hence, if we define

$$
V_{C}:=V_{C}^{\prime} \cup V_{C}^{\prime \prime}
$$

then

$$
V_{P}^{\prime} \cap V_{P}^{\prime \prime} \stackrel{(48)}{=} U \cap V_{C}^{\prime}=U \cap V_{C}=U \cap V_{C}^{\prime \prime}
$$

and it follows that

$$
G^{\prime} \backslash V_{C}=G^{\prime} \backslash V_{C}^{\prime}, \quad G^{\prime \prime} \backslash V_{C}=G^{\prime \prime} \backslash V_{C}^{\prime \prime}
$$

and

$$
V_{P} \backslash V_{C}=\left(V_{P}^{\prime} \backslash V_{C}\right) \uplus\left(V_{P}^{\prime \prime} \backslash V_{C}\right)=\left(V_{P}^{\prime} \backslash V_{C}^{\prime}\right) \uplus\left(V_{P}^{\prime \prime} \backslash V_{C}^{\prime \prime}\right) .
$$

Therefore,

$$
\begin{aligned}
G^{\ell} \backslash V_{C} \backslash V_{P}= & \left(\left(G^{\prime \ell^{\prime}}\right) \cup\left(G^{\prime \prime \ell^{\prime \prime}}\right)\right) \backslash V_{C} \downarrow\left(V_{P} \backslash V_{C}\right) \\
\stackrel{(54)}{=} & \left(\left(G^{\prime \ell^{\prime}} \backslash V_{C}\right) \cup\left(G^{\prime \prime \ell^{\prime \prime}} \backslash V_{C}\right)\right) \downarrow\left(\left(V_{P}^{\prime} \backslash V_{C}^{\prime}\right) \uplus\left(V_{P}^{\prime \prime} \backslash V_{C}^{\prime \prime}\right)\right) \\
\stackrel{(53)}{=} & \left(\left(G^{\prime \ell^{\prime}} \backslash V_{C}^{\prime}\right) \cup\left(G^{\prime \prime \ell^{\prime \prime}} \backslash V_{C}^{\prime \prime}\right)\right) \downarrow\left(V_{P}^{\prime} \backslash V_{C}^{\prime}\right) \downarrow\left(V_{P}^{\prime \prime} \backslash V_{C}^{\prime \prime}\right) \\
= & \left(G^{\prime \ell^{\prime}} \backslash V_{C}^{\prime} \downarrow\left(V_{P}^{\prime} \backslash V_{C}^{\prime}\right)\right) \cup\left(G^{\prime \prime \ell^{\prime \prime}} \backslash V_{C}^{\prime \prime} \downarrow\left(V_{P}^{\prime \prime} \backslash V_{C}^{\prime \prime}\right)\right) \\
= & \left(G^{\prime \ell^{\prime}} \backslash V_{C}^{\prime} \downarrow V_{P}^{\prime}\right) \cup\left(G^{\prime \prime \ell^{\prime \prime}} \backslash V_{C}^{\prime \prime} \downarrow V_{P}^{\prime \prime}\right),
\end{aligned}
$$

and, based on an induction argument, the last obtained term indicates the paintability of $G^{\ell} \backslash V_{C} \downarrow V_{P}$. However, this means that $V_{C}$ is good in $V_{P}$ with respect to the examined graph $G^{\ell}$.

Now, we are prepared to strengthen Thomassen's Theorem [Th], [Di, p. 122] about the 5-list colorability of planar graphs:

Theorem 2.6. Planar graphs are 5-paintable.

Proof. The proof works almost exactly the same as the original one, but the coloring extension arguments have to be replaced (see also [Di, p. 122]). We start with a slightly modified induction hypothesis, and will prove by induction the following assertion for all plane graphs $G$ with at least 3 vertices. In connection with Lemma 2.2 (which allows us to reinsert the removed edge $v_{1} v_{2}$ ) this assures the 5 -paintability of plan triangulations, and hence all planar graphs. The induction hypothesis reads as follows:

Suppose that every inner face of $G^{\ell}$ is bounded by a triangle and its outer face by a cycle $C=v_{1} \ldots v_{k} v_{1}$. Suppose further that there is no eraser at $v_{1}$ 
and at $v_{2}\left(\ell_{v_{1}}=\ell_{v_{2}}:=1\right)$, that there are 2 erasers at each other vertex $v_{i}$ of the boundary $C\left(\ell_{v_{i}}:=3\right)$, and that there are 4 at each inner vertex $u$ $\left(\ell_{u}:=5\right)$. Then Mrs. Correct can enforce a proper coloring of $G^{\ell} \backslash v_{1} v_{2}$.

If $|G|=3$, then $G=C$ and the assertion is trivial. We may thus assume that there are edges inside $C$, and we can distinguish between the following two cases:

Case 1. If $C$ has a chord $v_{i} v_{j}$, then $v_{i} v_{j}$ lies on two unique cycles

$$
C^{\prime}, C^{\prime \prime} \subseteq C+v_{i} v_{j}
$$

with

$$
v_{1} v_{2} \in C^{\prime} \text { and } v_{1} v_{2} \notin C^{\prime \prime} .
$$

Let $G^{\prime}$ resp. $G^{\prime \prime}$ denote the subgraph of $G$ induced by the vertices lying on or inside $C^{\prime}$ resp. $C^{\prime \prime}$. Using an induction argument, we know that the assertion holds for $G^{\prime \ell^{\prime}}$, with the inherited erasers $\left(\ell^{\prime}:=\left.\ell\right|_{\left.V\left(G^{\prime}\right)\right)}\right)$. Similarly, it also holds for $G^{\prime \prime}$, but with $v_{i}$ and $v_{j}$ in the place of $v_{1}$ and $v_{2}$, i.e., $G^{\prime \prime} \backslash v_{i} v_{j}$ is $\ell^{\prime \prime}$-paintable when all erasers at $v_{i}$ and at $v_{j}$ are removed $\left(\ell_{v_{i}}^{\prime \prime}=\ell_{v_{j}}^{\prime \prime}:=1\right.$ and $\ell_{u}^{\prime \prime}:=\ell_{u}$ for the other vertices $u$ in $\left.G^{\prime \prime}\right)$. Now Lemma 2.5 applies and proves the paintability of

$$
G^{\ell} \backslash v_{1} v_{2}=G^{\prime \ell^{\prime}} \backslash v_{1} v_{2} \cup G^{\prime \prime \ell^{\prime \prime}} \backslash v_{i} v_{j}
$$

Case 2. If $C$ has no chord, let $v_{1}, u_{1}, u_{2}, \ldots, u_{m}, v_{k-1}$ be the neighbors of $v_{k}$ in their natural cyclic order around $v_{k}$. By definition of $C$, all these neighbors $u_{i}$ lie in the inner face of $C$. Since the inner faces of $G$ are bounded by triangles, and there are no multiple edges,

$$
P:=v_{1} u_{1} \ldots u_{m} v_{k-1}
$$

is a path in $G$. Since $C$ is chordless,

$$
\tilde{C}:=P \cup\left(C \backslash v_{k}\right)
$$

is a cycle - the boundary cycle of $G \backslash v_{k}$. By induction we know that $G \backslash v_{k} \backslash v_{1} v_{2}$ is paintable, where at the new boundary vertices $u_{i}$ two erasers suffice.

We now extend the paintability of $G \backslash v_{k} \backslash v_{1} v_{2}$ to $G \backslash v_{1} v_{2} \backslash v_{k} v_{1}$ and finally to $G \backslash v_{1} v_{2}$. To this end we apply Lemma 2.4 to $G \backslash v_{1} v_{2} \backslash v_{k} v_{1}$, with $v_{k}$ in the role of $w$ and $v_{k-1}$ in the role of $u_{0}$. Afterwards, we apply Lemma 2.2, with $v_{k}$ in the role of $w$ and $v_{1}$ in the

role of $u$. Altogether, we had to add 2 erasers at each of the $u_{i}$ and on the new vertex $v_{k}$; the sizes of the other stacks remained unchanged.

\section{Kernels and Edge Paintability}

In this section we generalize some results about edge list colorability to edge paintability; where a graph $G$ is called edge $\ell$-paintable if its line graph is $\ell$-paintable. Two further edge paintability results, concerning the complete graph $K_{n}$ and regular planar graphs, 
will be presented in [Scha2]. All results of this section are based on the existence of kernels (Lemma 3.1) and the examination of orientations. We use the following notations for these kind of investigations:

$\rightarrow: E \longrightarrow V, e \longmapsto e^{\rightarrow}$ denotes a fixed orientation of $G$. Therefore, $\vec{e}$ is always one end of $e$, and $e^{\leftarrow}$ denotes the other one $\left(\left\{e^{\vec{r}}, e^{\leftarrow}\right\}=e\right) . \quad \vec{G}:=(V, E, \rightarrow)$ is the corresponding oriented graph. $D=D(G)=D(\vec{G})$ denotes the set of all orientations $\varphi: E \ni e \longmapsto e^{\varphi} \in e$ of $G$. We write $u \rightarrow v$ (resp. $u \stackrel{\varphi}{\rightarrow} v$ ) if we want to say that $u v \in E$ and that $(u v)^{\rightarrow}=v$ (resp. $\left.(u v)^{\varphi}=v\right) . \quad N_{\varphi}^{+}(v):=\{w \in V \quad v \stackrel{\varphi}{\rightarrow} w\}$ denotes the set of $\varphi$-successors of $v \in V, d_{\varphi}^{+}(v):=\left|N_{\varphi}^{+}(v)\right|$ its $\varphi$-outdegree, and $d_{\varphi}^{+}:=\left(d_{\varphi}^{+}(v)\right)_{v \in V}$ the outdegree tuple. We abbreviate $N^{+}(v):=N_{\rightarrow}^{+}(v)$ and $d^{+}:=d_{\rightarrow}^{+}$. Similarly, we define $N(v)=N_{G}(v):=\{w \in V \quad$ | $v w \in E\}$ and $d_{G}:=(d(v))_{v \in V}$. As usual, $\Delta(G)$ is the maximal degree, and $\Delta^{+}(\varphi)$ is the maximal outdegree of the vertices in $G$.

Now, the following paintability version of Bondy, Boppana and Siegel's Lemma, in [Ga, Lemma 2.1] or [Di, Lemma 5.4.3], follows easily with a simple induction argument from Definition 1.8:

Lemma 3.1 (Kernel Lemma). Let $\vec{G}$ be a directed graph, such that each induced subgraph $G\left[V_{P}\right]$ of $\vec{G}$ has a kernel - i.e., an independent subset $V_{C} \subseteq V_{P}$ such that, for each vertex $u \in V_{P} \backslash V_{C}$ there is a $\bar{u} \in V_{C}$ with $u \rightarrow \bar{u}$ - then $G$ is $\left(d^{+}+1\right)$-paintable.

Proof. We may assume $G \neq \varnothing$. Let $V_{C}$ be a kernel of a fixed given nonempty subset $V_{P} \subseteq V$. As necessarily $V_{C} \neq \varnothing$, and as $G \backslash V_{C}$ fulfills the preconditions of the Lemma, we may apply an induction argument, and see that $G \backslash V_{C}$ is $\left(d_{G \backslash V_{C}}^{+}+1\right)$-paintable, i.e.,

$$
\left(G \backslash V_{C}\right)^{d_{G \backslash V_{C}}^{+}+1+1_{\left(V_{P} \backslash V_{C}\right)}}\left\lfloor V_{P}=\left(G \backslash V_{C}\right)^{d_{G \backslash V_{C}}^{+}+1}\right.
$$

is paintable. Now, because of

$$
d_{G}^{+}(v)>d_{G \backslash V_{C}}^{+}(v) \quad \text { for all } v \in V_{P} \backslash V_{C},
$$

the paintability of

$$
G^{d_{G}^{+}+1} \backslash V_{C} \downarrow V_{P}
$$

follows; so that the recursive Definition 1.8 applies.

Galvin used in [Ga] Bondy, Boppana and Siegel's Lemma to prove the list coloring conjecture for bipartite graphs (see also [Di, Theorem 5.4.4]). Using our version this can be strengthened to paintability (without further modifications in the proof). Together with König's classical calculation [Di, Proposition 5.3.1] of the chromatic index of bipartite graphs we obtain:

Theorem 3.2. Bipartite graphs $G$ are edge $\Delta(G)$-paintable. 
Galvin's result also implies the existence of certain generalized Latin Squares, which was conjectured by Dinitz. With the stronger Theorem 3.2 this existence result can be generalized further, leading to a version with stacks of erasers on a „chess board“.

Borodin, Kostochka and Woodall exploited in [BKW] Galvin's remarkable new method to prove further sharpenings and applications. We strengthen their main result $[\mathrm{BKW}$, Theorem 3], and our Theorem 3.2, as follows:

Theorem 3.3. Bipartite multigraphs $G$ are edge $\ell$-paintable, when for each edge $e=u w$ we set

$$
\ell_{e}:=\max \{d(u), d(w)\}
$$

Proof. We refer to Galvin's original proof as it was printed in Diestel's book [Di]. Borodin, Kostochka and Woodall's proof use a terminology different from those in [Di, Theorem 5.4.4 \& Corollary 5.4.5], and does not explicitly work with orientations. However, the only real difference to the proof in [Di] is that the authors have chosen the underlying coloring $c: E \longrightarrow \mathbb{Z}$ more carefully (see the remark after [BKW, Corollary 1.1]). Based on the construction of $c$ in the proof of [BKW, Theorem 3], and using our strengthened Kernel Lemma 3.1 instead of [Di, Lemma 5.4.3], the proof in [Di] yields the stated theorem.

They also provide a proof for a strengthening of Shannon's bound of the chromatic index of multigraphs. This proof is based on the following interesting lemma, which we state for paintability:

Lemma 3.4. If $G, H$ and $B$ are multigraphs, where $B$ is bipartite and $G=H \cup B$, and if

$$
\ell_{e}:=\max \left\{d_{G}(u)+d_{H}(w), d_{H}(u)+d_{G}(w)\right\} \quad \text { for each edge } e=u w,
$$

then $G$ is edge $\ell$-paintable.

Proof. The proof is based on Theorem 3.3, and works almost exactly as in [BKW, Lemma 4.1]: We may assume

$$
E(H) \cap E(B)=\varnothing .
$$

Since $d_{G}(v) \geq d_{H}(v)$ for each $v \in V$, it follows that

$$
\ell_{e}>d_{L H}(e) \text { for all } e \in E,
$$

where $L H$ is the line graph of $H$. Hence, as a repeated application of the simple Cut Lemma 2.3 (with $|U|=1$ ) shows, $H$ is edge paintable using the inherited erasers.

Using Theorem 3.3, we see that the other part $B$ is edge $\ell^{\prime}$-paintable, where

$$
\ell_{u w}^{\prime}:=\max \left\{d_{B}(u), d_{B}(w)\right\} \quad \text { for all } u w \in E(B) .
$$

The ELECtronic Journal of COMbinatorics 16 (2009), \#R77 
Since each edge $u w$ of $B$ (as a vertex of the line graph $L G$ ) has

$$
\eta_{u w}:=\left|N_{L G}(u w) \cap E(H)\right|=d_{H}(u)+d_{H}(w)
$$

neighbors in $E(H)$, so that

$$
\begin{aligned}
\ell_{u w} & =\max \left\{d_{G}(u)+d_{H}(w), d_{H}(u)+d_{G}(w)\right\} \\
& =\max \left\{d_{B}(u)+\left(d_{H}(u)+d_{H}(w)\right),\left(d_{H}(u)+d_{H}(w)\right)+d_{B}(w)\right\} \\
& =\max \left\{d_{B}(u), d_{B}(w)\right\}+\left(d_{H}(u)+d_{H}(w)\right) \\
& =\ell_{u w}^{\prime}+\eta_{u w},
\end{aligned}
$$

the Cut Lemma2.3 (with $L G, E(B), E(H)$ in the place of $G, U, W$ ) to prove the $\ell$-paintability of $G$.

With this lemma we obtain the following strengthening of Shannon's bound:

Theorem 3.5. Multigraphs $G$ are edge $\ell$-paintable, where

$$
\ell_{u w}:=\max \{d(u), d(w)\}+\left\lfloor\frac{1}{2} \min \{d(u), d(w)\}\right\rfloor \quad \text { for all } u w \in E .
$$

In particular, $G$ is edge $\left\lfloor\frac{3}{2} \Delta(G)\right\rfloor$-paintable.

Proof. As in [BKW, Theorem 4] one can apply Lemma 3.4 to a maximal cut

$$
B=(V, E(U, W)), \quad V=U \uplus W
$$

in $G$, and to

$$
H:=G \backslash E(B)
$$

which fulfills

$$
d_{H}(v) \leq \frac{1}{2} d_{G}(v) \text { for all } v \in V
$$

since otherwise we could move a vertex $v$ to the other side of the partition, and would obtain a contradiction to the maximality of $|E(U, W)|$.

The figure $\left\lfloor\frac{3}{2} \Delta(G)\right\rfloor$ in this theorem is best possible. The so-called "thick triangle" with $\left\lfloor\frac{1}{2} \Delta\right\rfloor,\left\lfloor\frac{1}{2} \Delta\right\rfloor$ and $\left\lceil\frac{1}{2} \Delta\right\rceil$ edges between the vertices shows this; it has chromatic index $\left\lfloor\frac{3}{2} \Delta\right\rfloor$.

Clearly, it would be interesting to find a paintability version of Vizing's Theorem. This is an open problem, even for list colorings. The recoloring techniques (Kempe-chains) used in the known proofs of Vizing's Edge Coloring Theorem do not work with list colorings. In [Ko] Kostochka needed the additional assumption that $G$ has girth at least $8 \Delta(G)(\ln (\Delta(G))+1.1)$, in order to prove that simple graphs $G$ are edge $(\Delta(G)+1)$-list colorable. However, if the list color conjecture is true, this holds without further assumptions about the girth as well. 


\section{Acknowledgement:}

We are grateful to Melanie Win Myint, Stefan Felsner, Anton Dochtermann, Caroline Faria and Stephen Binns for helpful comments. Furthermore, the author gratefully acknowledges the support provided by the King Fahd University of Petroleum and Minerals, the Technical University of Berlin and the Eberhard Karls University of Tübingen during this research.

\section{References}

[Al] N. Alon: Restricted Colorings of Graphs. In "Surveys in combinatorics, 1993", London Math. Soc. Lecture Notes Ser. 187, Cambridge Univ. Press, Cambridge 1993, 1-33.

[Al2] N. Alon: Combinatorial Nullstellensatz.

Combin. Probab. Comput. 8, No. 1-2 (1999), 7-29.

[AlTa] N. Alon, M. Tarsi: Colorings and Orientations of Graphs. Combinatorica 12 (1992), 125-134.

[BGKZ] T. Bartnicki, J. Grytczuk, H. A. Kierstead, X. Zhu: The Map-Coloring Game. Am. Math. Monthly 114 No. 9 (2007), 793-803

[BKW] O. V. Borodin, A. V. Kostochka, D. R. Woodall:

List Edge and List Total Colourings of Multigraphs.

J. Combin. Theory Ser. B 71(2) (1997), 184-204.

[Co] J. H. Conway: On Numbers and Games. Academic Press, London (1976). Second edition: A. K. Peters, Wellesley/MA (2001).

[Di] R. Diestel: Graph Theory. (3rd edition) Springer, Berlin 2005.

[DnZh] T. Dinski and X. Zhu: Game Chromatic Number of Graphs. Discrete Math. 196 (1999), 109-115.

[Ga] F. Galvin: The List Chromatic Index of a Bipartite Multigraph. J. Combin. Theory (B) 63 (1995), 153-158.

[JeTo] T. R. Jensen, B. Toft: Graph Coloring Problems. Wiley, New York 1995.

[KTV] J. Kratochvíl, Zs. Tuza, M. Voigt:

New Trends in the Theory of Graph Colorings: Choosability and List Coloring. In: R.L. Graham et al., Editors, Contemporary Trends in Discrete Mathematics. DIMACS Ser. Discr. Math. Theo. Comp. Sci. 49, Amer. Math. Soc. (1999), 183-19\%.

[Ko] A. V. Kostochka: List Edge Chromatic Number of Graphs with Large Girth. Discrete Mathematics 101(1-3) (1992), 189-201.

[Scha1] U. Schauz: Algebraically Solvable Problems:

Describing Polynomials as Equivalent to Explicit Solutions The Electronic Journal of Combinatorics 15 (2008), \#R10. 
[Scha2] U. Schauz: Flexible Color Lists in Brooks' Theorem and in Alon and Tarsi's Theorem.

Submitted to the Electronic Journal of Combinatorics.

[Scha3] U.Schauz: A Paintability Version of the Combinatorial Nullstellensatz, and List Colorings of $k$-partite $k$-uniform Hypergraphs.

Submitted to the Electronic Journal of Combinatorics.

[SSt] D. Schleicher, M. Stoll: An Introduction to Conway's Numbers and Games. http://arxiv.org/abs/math.CO/0410026

[Th] C. Thomassen: Every Planar Graph is 5-Choosable.

J. Combinatorial Theory B 62 (1994), 180-181.

[Tu] Zs. Tuza: Graph Colorings With Local Constraints - A Survey.

Discuss. Math. Graph Theory, 17, (1997), 161-228. 